\title{
Re-evaluation of the Taiwanese white-toothed shrew, Crocidura tadae Tokuda and Kano, 1936 (Insectivora: Soricidae) from Taiwan and two offshore islands
}

\author{
Yin-Ping Fang and Ling-Ling Lee* \\ Department of Zoology, National Taiwan University, Taipei, Taiwan, ROC, 10617 \\ (Accepted 16 May 2001)
}

\begin{abstract}
Crocidura tadae was first reported as a new species from Orchid Island by Tokuda \& Kano (1936). However, both $C$. tadae and C. kurodai from Taiwan were considered as subspecies of $C$. horsfieldii by subsequent researchers. In the present study, the karyotypic and morphological differences among the potential C. tadae specimens from Taiwan main island and two offshore islands (Orchid Island and Green Island) were examined. The results revealed that there is no significant difference in the karyotypes of these shrews but that their karyotype is different from that of other Crocidura species. Therefore, the Tadae's shrew C. tadae Tokuda \& Kano, 1936 should be a valid species. Furthermore, morphological variation in 25 external and cranial characters of 106 specimens were analysed using univariate statistics, and eight selected characters were used for multivariate analyses. Univariate statistical comparisons of the three insular samples revealed that shrews of the Green Island population were the largest in most measurements of characters and shrews of the Orchid Island population were the smallest. Multivariate analysis differentiated the Taiwan samples from the other two insular samples. Therefore, three subspecies of C. tadae are recognized: C. t. tadae (Orchid Island); C. t. kurodai (Taiwan) and C. t. lutaoensis subsp. nov (Green Island).
\end{abstract}

Key words: Crocidura tadae, karyotype, morphometrics, new subspecies

\section{INTRODUCTION}

Tokuda \& Kano (1936) first reported a new species of white-toothed shrew, Crocidura tadae, from Orchid Island (i.e. Lanyu or Kotôshô, south-east offshore island of Taiwan). Kuroda (1952), however, treated C. tadae as a subspecies of $C$. horsfieldii (Tomes, 1856), and noted that this subspecies is not found on the main island of Taiwan. Later, Jones, Lim \& Cross (1971) reported an unknown species of Crocidura from Taiwan. Jameson \& Jones (1977) later described this form as a new subspecies C. h. kurodai on the basis of three specimens from Linkou (north Taiwan) and Nantou Hsien (central Taiwan). They reported that the body size of kurodai was larger than that of tadae, but included both kurodai and tadae in C. horsfieldii complex. Other authors, including Jenkins (1976), Corbet \& Hill (1992) and Hutterer (1993), have mostly followed this classification, and treated kurodai and tadae as synonyms of $C$. horsfieldii.

*All correspondence to: L.-L. Lee

E-mail: leell@ccms.ntu.edu.tw
However, Fang et al. (1997) re-evaluated C. h. kurodai as a unique species, C. kurodai, on the basis of differences in karyotype and morphology between samples from central Taiwan and south India. Chromosome numbers and the fundamental number of C. kurodai and C. horsfieldii are reported as 40 and 54 (Fang et al., 1997), and 38 and 48 (Rao \& Aswathanarayana, 1978), respectively. Meanwhile, samples of tadae have been too few for reasonable analysis. Therefore, the taxonomy of kurodai and tadae remains uncertain.

Recently, a modified system of pitfall traps was used in combination with drift fences for capturing live shrews. This trapping system proves to be more efficient in collecting live Crocidura shrews than box traps. We were able to obtain large samples of Crocidura shrews from Taiwan main island and two offshore islands, i.e. Orchid Island and Green Island (i.e. Lutao, Fig. 1), and provide the first record of white-toothed shrews (genus Crocidura) from Green Island.

The purposes of this study were to examine karyotypes of these crocidura shrews, the morphological difference among these three insular populations, and to analyse the geographical variation of these shrews by 


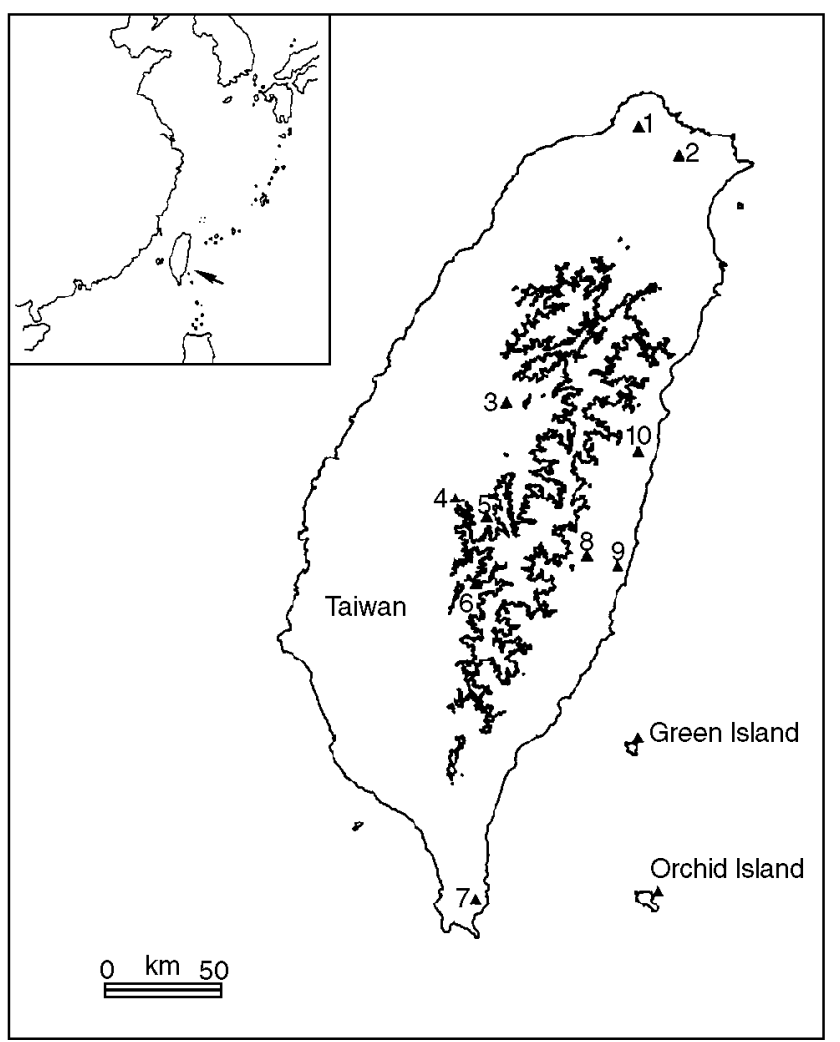

Fig. 1. Localities of Crocidura tadae specimens collected for this study: 1, Erhtzuping; 2, Hsichih; 3, Huisun; 4, Hsitou; 5, Tungpu; 6, Meilan; 7, Nanjenshan; 8, Chungping; 9, Chihkeshan; 10, Shuilien and two offshore islands, Green Island (Lutao) and Orchid Island (Lanyu). Contour line, altitudes of $2000 \mathrm{~m}$ a.s.1.

multivariate approaches. Based on these results, the taxonomy of the Crocidura species complex from these three insular populations was re-evaluated.

\section{MATERIALS AND METHODS}

\section{Field methods}

Shrews were collected between 1996 and 1999 from 10 localities in Taiwan main island and 2 offshore islands, i.e. Green Island and Orchid Island (Fig. 1). Green Island, with a total area of $15.1 \mathrm{~km}^{2}$, is located $c .33 \mathrm{~km}$ south-east of Taiwan (i.e. $22^{\circ} 38^{\prime} \mathrm{N}, 121^{\circ} 28^{\prime} \mathrm{E}$ ). Orchid Island, with a total area of $46.8 \mathrm{~km}^{2}$, is located c. $90 \mathrm{~km}$ SE of Taiwan (i.e. $22^{\circ} 03^{\prime} \mathrm{N}, 121^{\circ} 33^{\prime} \mathrm{E}$ ).

The animals were captured in pitfall traps. Arrays of 4-7 pitfall traps (plastic flower pots, $15 \mathrm{~cm}$ in diameter, $21 \mathrm{~cm}$ deep with a hole in the bottom to drain water) and drift-fences (1-2 $\mathrm{m}$ long) were arranged in Y-shape or T-shape, depending on the terrain. A detailed account of a similar design can be found in Kirkland \& Sheppard (1994). To keep the animals alive, mealworms (larvae of Tenebrio sp.) were put in the pitfalls. We also put shredded coconut along the drift-fences to enhance the capture rate.
Pitfall traps were set for $4-6$ consecutive nights at each locality. Other small mammals captured at each locality and surrounding vegetation were also recorded. Most live shrews were brought back to the laboratory for karyotyping, and all the specimens were measured and prepared for morphological study. All the specimens are deposited in the Department of Zoology, National Taiwan University (NTU), Taipei.

\section{Karyotype}

Metaphase spreads were prepared from bone marrow cells of yeast-stimulated individuals (Lee \& Elder, 1980) and stained with $5 \%$ Giemsa stain. The method is outlined in Benn \& Perle (1986). The diploid number (2n) and fundamental number (FN) including female sex chromosomes (XX) were determined by examining at least 30 complete metaphase cells from each animal. Chromosome morphology was categorized according to that of Ruedi \& Vogel (1995). Biarmed chromosomes with subequal arms were considered as metacentric (M), and those with arms of unequal lengths as submetacentric (SM). When the short arm is $>50 \%$ of the length of the long one, the chromosome was regarded as subtelocentric (ST), and if only 1 pair of chromosomal arms was visible, as acrocentric (A). The G-band techniques of Seabright (1972) were also applied.

\section{Morphology}

The external, cranial and mandibular characters of the shrews were examined by conventional methods. The measurements of body size were taken from freshly killed animals. Most cranial and mandibular measurements were taken following the characters selected by Kitchener, Hisheh et al. (1994), Motokawa et al. (1996) and Ruedi (1995). For informative comparison, some other measurements were also taken following Tokuda \& Kano (1936) and Jameson \& Jones (1977).

Seven external, 18 cranial and mandibular characters (Fig. 2) were measured. These measurements were: head to vent length (HV); tail to vent length (TV); the ratio of TV and HV (TH); hind foot length (HF: exclusive of claw); ear length (EL); distribution of vibrissae over the length of the tail (VL); the ratio of VL and TL (VT); greatest length of skull (GLS); condylobasal length (CL); postpalatal length (PPL); upper tooth row length (IM3S); greatest width at the second molar (M2B); rostral breadth (ROB); palatal width at the third molars (M3B); length of upper molariform teeth (PM3); braincase breath (BB); interorbital breadth (IOB); condyle to glenoid length (CTG); postpalatal depth (PPD); rostral length (ROL); lower tooth row length excluding first incisor (IM3I); mandibular length (MAL); mandibular height at coronoid process $(\mathrm{MH})$; tip of coronoid process to the end of angular process length (ML), angular process length (APL). To reduce any discrepancies, all measurements were taken by the first author. 

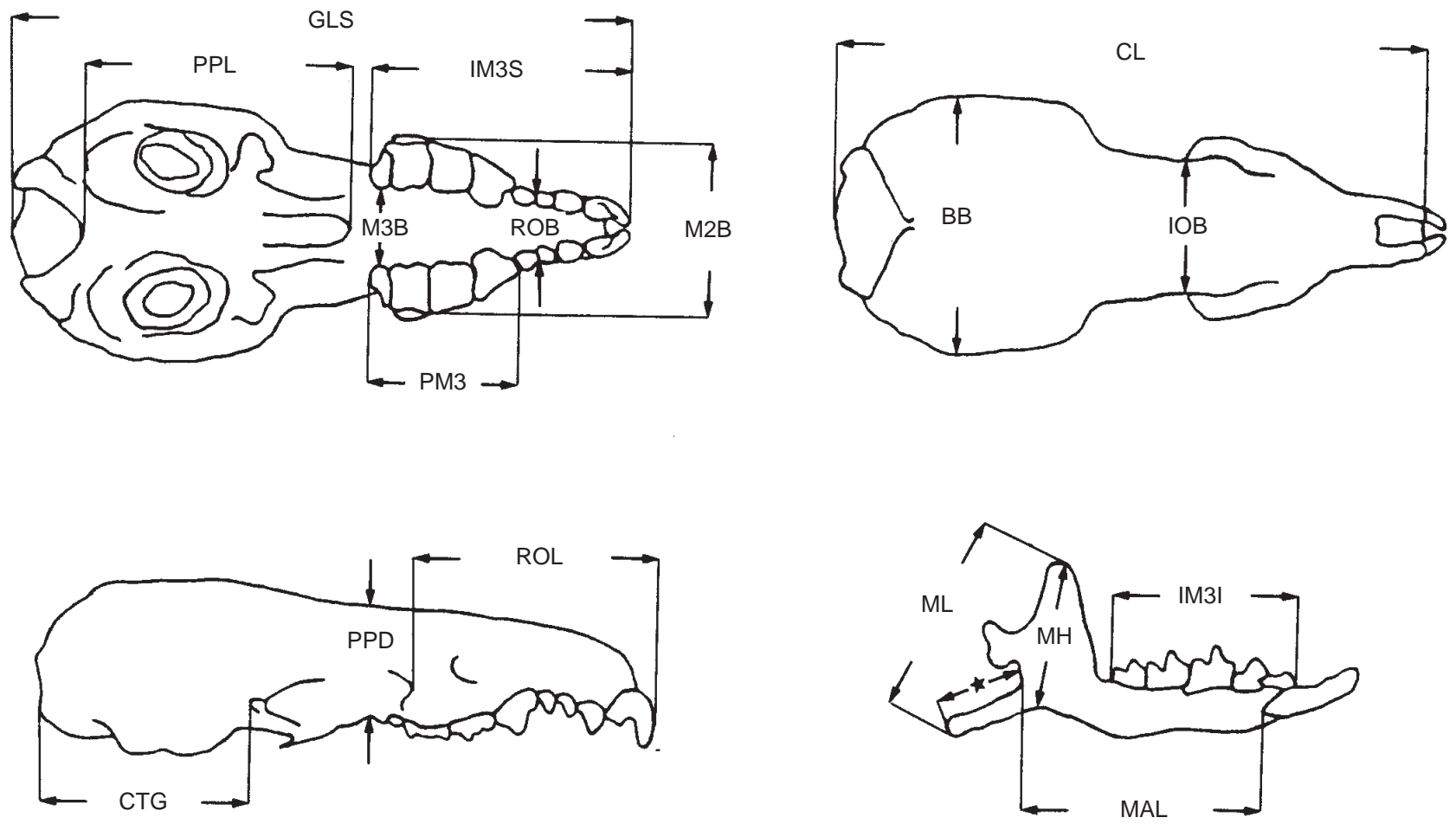

Fig. 2. Measurement points for cranial and mandibular characters of Crocidura tadae. See text for abbreviations of character variables. *, angular process length (APL).

External characters were measured with a metric calliper graduated to $0.1 \mathrm{~mm}$, and skull characters were measured with a digimatic calliper graduated to $0.01 \mathrm{~mm}$.

Sexual maturity was used as our criterion of adult status. Males with spermatozoa were classified as sexually mature. Females were divided into 2 reproductive categories on the basis of their uterine and nipple condition: non-breeding and breeding (both pregnant and lactating). Because no juvenile samples were collected, all samples were used in the following analysis.

To compare the differences in the measurements of the external, cranial and mandibular characters among 3 insular groups, i.e. Taiwan, Green Island, and Orchid Island, the samples from Taiwan were first clustered into a single group. Student's $t$-test was used to examine sexual differences in the measurements of the above characters. Then, ANOVA was run to examine the relationship between all the characters and 2 factors, sex and island. Finally, Tukey's multiple comparison was used to investigate the differences among the 3 insular populations.

Canonical discriminate analysis (CDA) was performed on all 25 skull and external characters to examine the degree of separation of the 3 insular groups. All measurements were log-transformed before CDA. However, to get a better separation of these groups with a minimum number of variables and to adapt to the smallest sample size of island population, fewer characters (8) were used in the final analyses. All statistical analyses were run on a PC computer using SYSTAT 7.0 programs (SPSS, 1997).

\section{RESULTS}

A total of 106 specimens of C. kurodai and C. tadae was collected from Green Island $(n=15)$, Orchid Island $(n=19)$ and 10 localities of Taiwan $(n=72)$ (Fig. 1). The trapping records indicated that $C$. kurodai is distributed in Taiwan at elevations from sea level to $2000 \mathrm{~m}$, and the dominant habitat is secondary forest at elevations from 500 to $1500 \mathrm{~m}$. The capture rates of Crocidura at each locality varied and so did the shrew species living sympatrically. In Taiwan, C. kurodai often live sympatrically with other shrews such as Crocidura attenuata Milne-Edwards, 1872; Anourosorex squamipes MilneEdwards, 1872; and Soriculus sodalis (Thomas, 1913). However, there is only one coexisting shrew, Suncus murinus (L.), on Green Island, and no other shrew was found on Orchid Island except $C$. tadae.

Sex ratios of captured shrews also varied at different localities. While the sex ratio was strongly biased toward males in Taiwan $\left(>3: 1, \chi^{2}=26.89, P<0.001\right)$, the bias was not significant on the two offshore islands (c. $1: 1, \chi^{2}=0.067$ Green Island, 0.473 Orchid Island, $P>0.05)$.

\section{Karyotype}

Twenty-four shrews were karyotyped. More than two males and two females were examined for each insular group. The results of the conventional karyotypes are shown in Fig. 3. The diploid chromosome number of 24 


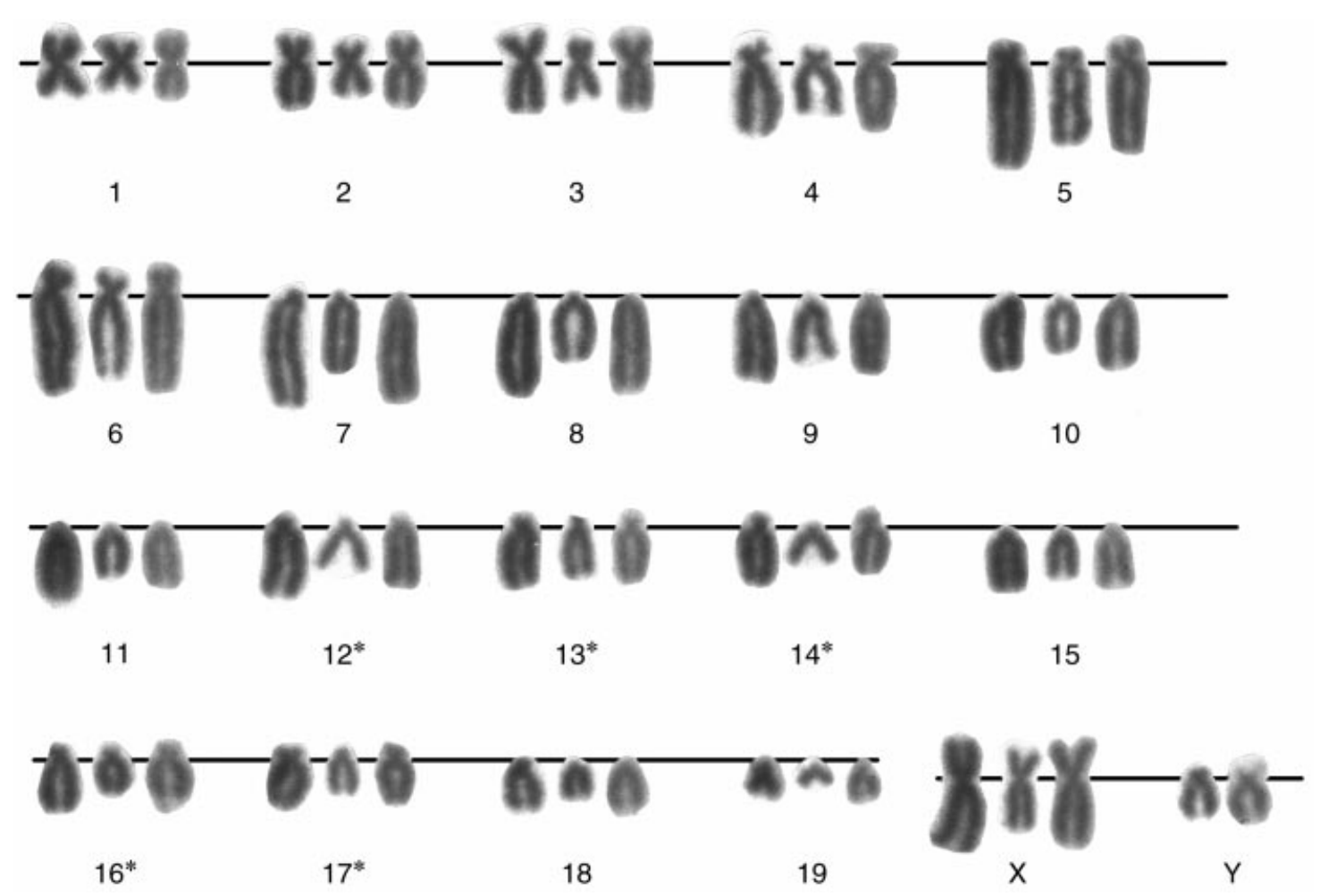

Fig. 3. The conventional karyotypes of Crocidura tadae from Orchid Island and Taiwan. Three parallel chromosomes arranged in following order: FS4104 (Orchid Island, female), FS4401 (Meilan, Taiwan, male) and FS3103 (Hsitou, Taiwan, male). *, variation of chromosome condensation in the short arm of the 12th, 13th, 14th, 16th and 17th pairs of chromosome.

shrews was invariably determined as 40 . However, fundamental numbers were determined as either 54 or 64. This was a result of the difference in the degree of chromosome condensation. Five pairs of chromosomes could not be distinguished clearly as being subtelocentric (ST), or acrocentric (A) (Fig. 3). The standard karyotypes of C. kurodai reported by Fang et al. (1997) showed the same problem, i.e. the 12th, 13th, 14th, 16th and 17th pairs of chromosome have condensed short arms but the chromatids did not separate. If these chromosomes were considered as subtelocentrics (ST), the fundamental numbers of $C$. kurodai would change from 54 to 64 .

Because the karyotypic patterns of different specimens were similar, and G-band analysis revealed no significant karyotype variation among the insular populations, we, therefore, consider C. kurodai and C. tadae as synonyms. The previous name for this valid species is C. tadae Tokuda \& Kano, 1936, which can be separated from $C$. horsfieldii on the basis of chromosomal characteristics.

\section{Morphology}

\section{Univariate analysis}

External, cranial and mandibular measurements of samples from the three insular groups are presented in
Table 1. Measurements of males and females are presented separately, because about one-third of the 25 characters showed significant sexual differences within each insular group. However, the characters that showed sexual dimorphism were different for each insular group. In the Taiwan group, most dimorphism appeared in cranial and mandibular characters $(P<0.05)$, and highly significant dimorphism $(P<0.01)$ was found in PM3. In the Green Island group, the dimorphism distributed more evenly in external and skull characters, and highly significant dimorphism was found in TL, IM3S, and ROL. Measurements of most characters are larger in males than in females on Green Island and Orchid Island, but the opposite was true on Taiwan.

Multiple regression analysis for all 25 characters vs sex and island revealed that only five of these 25 characters showed significant correlation with sex alone $(P<0.05$, Table 2$)$, but 23 of 25 characters were highly correlated with islands $(P<0.01)$. The results strongly supported island rather than sexual correlation. Therefore, we pooled data of males and females for each insular group in the following analyses.

Comparisons of shrews from three island groups also revealed that of the 25 characters, shrews of the Green Island population were the largest in most measurements and the shrews of the Orchid Island population were smallest. Shrews of the Taiwan populations were intermediate in average size (Table 3), although the range (min-max) of each character was always broader 
Table 1. Measurements (mm) for external, cranial, mandibular, characters of adult male (M) and female (F) Crocidura tadae from Taiwan, Green Island and Orchid Island (see Materials and methods for definition of character codes). Sample size in parenthesis; SD, standard deviation. Significance levels: *, $0.05>P>0.01 ; * *, 0.01>P>0.001$

\begin{tabular}{|c|c|c|c|c|c|c|c|c|c|c|}
\hline \multirow[b]{2}{*}{ Character } & & \multicolumn{3}{|c|}{ Taiwan } & \multicolumn{3}{|c|}{ Green Island } & \multicolumn{3}{|c|}{ Orchid Island } \\
\hline & & $\mathrm{F}(14)$ & $\mathrm{M}(58)$ & $P$ & $\mathrm{~F}(7)$ & $\mathrm{M}(8)$ & $P$ & $\mathrm{~F}(8)$ & $\mathrm{M}(11)$ & $P$ \\
\hline \multicolumn{11}{|l|}{ External } \\
\hline \multirow{2}{*}{ HV } & Mean & 61.69 & 62.62 & & 61.81 & 64.60 & & 58.14 & 61.26 & \\
\hline & SD & 3.31 & 3.19 & & 4.40 & 2.73 & & 3.19 & 2.62 & $*$ \\
\hline \multirow{2}{*}{$\mathrm{TV}$} & Mean & 49.05 & 48.61 & & 49.04 & 53.50 & & 43.53 & 47.35 & \\
\hline & SD & 3.98 & 3.75 & & 2.35 & 2.53 & $* *$ & 4.37 & 1.57 & $*$ \\
\hline \multirow[t]{2}{*}{$\mathrm{TH}$} & Mean & 0.80 & 0.78 & & 0.80 & 0.83 & & 0.75 & 0.77 & \\
\hline & SD & 0.09 & 0.06 & & 0.03 & 0.03 & $*$ & 0.08 & 0.04 & \\
\hline \multirow[t]{2}{*}{$\mathrm{HF}$} & Mean & 11.74 & 11.93 & & 11.99 & 12.39 & & 11.71 & 11.54 & \\
\hline & SD & 0.24 & 0.53 & & 0.44 & 0.29 & & 0.28 & 0.31 & \\
\hline \multirow[t]{2}{*}{ EL } & Mean & 8.46 & 8.19 & & 8.50 & 8.64 & & 7.74 & 8.22 & \\
\hline & SD & 0.33 & 0.50 & $*$ & 0.37 & 0.39 & & 0.50 & 0.24 & $*$ \\
\hline \multirow[t]{2}{*}{ VL } & Mean & 19.73 & 19.99 & & 18.46 & 20.89 & & 13.05 & 15.25 & \\
\hline & SD & 2.82 & 3.45 & & 7.28 & 2.53 & $*$ & 1.45 & 1.99 & $*$ \\
\hline \multirow[t]{2}{*}{ VT } & Mean & 0.40 & 0.41 & & 0.38 & 0.39 & & 0.30 & 0.32 & \\
\hline & $\mathrm{SD}$ & 0.04 & 0.06 & & 0.03 & 0.05 & & 0.03 & 0.30 & \\
\hline \multicolumn{11}{|l|}{ Cranial } \\
\hline \multirow[t]{2}{*}{ GLS } & Mean & 17.91 & 17.64 & & 17.81 & 18.41 & & 17.10 & 17.35 & \\
\hline & SD & 0.38 & 0.42 & $*$ & 0.44 & 0.23 & $*$ & 0.42 & 0.36 & \\
\hline \multirow[t]{2}{*}{ CL } & Mean & 17.14 & 16.89 & & 17.16 & 17.64 & & 16.34 & 16.66 & \\
\hline & SD & 0.34 & 0.43 & $*$ & 0.59 & 0.29 & & 0.42 & 0.37 & \\
\hline \multirow{2}{*}{ PPL } & Mean & 8.00 & 7.95 & & 7.94 & 8.19 & & 7.63 & 7.74 & \\
\hline & SD & 0.19 & 0.22 & & 0.39 & 0.13 & & 0.23 & 0.14 & \\
\hline IM3S & Mean & 7.74 & 7.58 & & 7.73 & 8.01 & & 7.44 & 7.52 & \\
\hline & SD & 0.20 & 0.22 & $*$ & 0.12 & 0.12 & $* *$ & 0.16 & 0.18 & \\
\hline M2B & Mean & 5.25 & 5.25 & & 5.39 & 5.45 & & 5.12 & 5.21 & \\
\hline & SD & 0.09 & 0.11 & & 0.15 & 0.12 & & 0.11 & 0.11 & \\
\hline ROB & Mean & 2.19 & 2.17 & & 2.23 & 2.29 & & 2.12 & 2.19 & \\
\hline & SD & 0.07 & 0.07 & & 0.10 & 0.08 & & 0.04 & 0.06 & $*$ \\
\hline M3B & Mean & 2.12 & 2.10 & & 2.16 & 2.22 & & 2.09 & 2.14 & \\
\hline & SD & 0.07 & 0.08 & & 0.09 & 0.04 & & 0.07 & 0.07 & \\
\hline PM3 & Mean & 4.50 & 4.38 & & 4.49 & 4.61 & & 4.30 & 4.34 & \\
\hline & SD & 0.12 & 0.14 & $* *$ & 0.11 & 0.05 & $*$ & 0.09 & 0.10 & \\
\hline $\mathrm{BB}$ & Mean & 8.06 & 8.05 & & 8.38 & 8.50 & & 8.06 & 8.26 & \\
\hline & SD & 0.18 & 0.21 & & 0.26 & 0.16 & & 0.17 & 0.17 & $*$ \\
\hline IOB & Mean & 3.92 & 3.86 & & 4.07 & 4.14 & & 3.81 & 3.89 & \\
\hline & SD & 0.11 & 0.14 & & 0.10 & 0.14 & & 0.09 & 0.09 & \\
\hline CTG & Mean & 7.79 & 7.74 & & 7.65 & 7.83 & & 7.43 & 7.57 & \\
\hline & SD & 0.16 & 0.23 & & 0.29 & 0.10 & & 0.21 & 0.14 & \\
\hline PPD & Mean & 3.28 & 3.27 & & 3.38 & 3.41 & & 3.23 & 3.28 & \\
\hline & SD & 0.12 & 0.09 & & 0.14 & 0.07 & & 0.07 & 0.08 & \\
\hline ROL & Mean & 6.81 & 6.63 & & 6.79 & 7.08 & & 6.49 & 6.53 & \\
\hline & SD & 0.20 & 0.20 & $*$ & 0.14 & 0.15 & $* *$ & 0.11 & 0.16 & \\
\hline Mandibula & & & & & & & & & & \\
\hline IM3I & Mean & 5.36 & 5.21 & & 5.39 & 5.52 & & 5.17 & 5.21 & \\
\hline & $\mathrm{SD}$ & 0.16 & 0.18 & $*$ & 0.10 & 0.05 & $*$ & 0.08 & 0.16 & \\
\hline MAL & Mean & 7.34 & 7.17 & & 7.51 & 7.67 & & 7.05 & 7.14 & \\
\hline & SD & 0.24 & 0.21 & $*$ & 0.30 & 0.29 & & 0.16 & 0.23 & \\
\hline MH & Mean & 4.05 & 4.12 & & 4.16 & 4.27 & & 3.93 & 3.96 & \\
\hline & SD & 0.14 & 0.16 & & 0.10 & 0.11 & & 0.07 & 0.11 & \\
\hline ML & Mean & 5.30 & 5.35 & & 5.35 & 5.56 & & 5.06 & 5.20 & \\
\hline & SD & 0.20 & 0.16 & & 0.18 & 0.19 & & 0.11 & 0.15 & $*$ \\
\hline APL & Mean & 1.97 & 2.01 & & 1.96 & 2.04 & & 1.91 & 2.03 & \\
\hline & SD & 0.10 & 0.11 & & 0.10 & 0.07 & & 0.14 & 0.06 & * \\
\hline
\end{tabular}


Table 2. Multiple regression on sex and island populations of Crocidura tadae (Taiwan, Green Island and Orchid Island) vs external, cranial, mandibular, and dental characters. $F$ values are presented for the main effects. For definition of character codes, see Materials and methods. Significance levels: *, $0.05>$ $P>0.01$; $^{*}, 0.01>P>0.001 ; * * *, P<0.001$

\begin{tabular}{|c|c|c|c|c|c|c|}
\hline \multirow[b]{2}{*}{ Character } & \multicolumn{2}{|c|}{ Sex } & \multicolumn{2}{|c|}{ Island } & \multicolumn{2}{|c|}{ Sex $\times$ island } \\
\hline & $F$-values & $P$ & $F$-values & $P$ & $F$-values & $P$ \\
\hline \multicolumn{7}{|l|}{ External } \\
\hline $\mathrm{HV}$ & 7.963 & $* *$ & 5.676 & $* *$ & 0.978 & \\
\hline TV & 8.607 & $* *$ & 11.783 & $* * *$ & 4.021 & $*$ \\
\hline $\mathrm{TH}$ & 0.599 & & 2.694 & & 1.558 & \\
\hline $\mathrm{HF}$ & 5.786 & $*$ & 12.157 & $* * *$ & 0.315 & \\
\hline EL & 1.079 & & 7.682 & $* *$ & 4.999 & $* *$ \\
\hline VL & 4.690 & $*$ & 25.509 & $* * *$ & 1.123 & \\
\hline VT & 1.282 & & 23.688 & $* * *$ & 0.079 & \\
\hline \multicolumn{7}{|l|}{ Cranial } \\
\hline GLS & 3.580 & & 21.307 & $* * *$ & 7.043 & $* *$ \\
\hline $\mathrm{CL}$ & 2.904 & & 19.893 & $* * *$ & 5.439 & $* *$ \\
\hline PPL & 3.569 & & 15.034 & $* * *$ & 2.560 & \\
\hline IM3S & 1.538 & & 15.121 & $* * *$ & 7.098 & $* *$ \\
\hline M2B & 3.197 & & 20.289 & $* * *$ & 1.268 & \\
\hline ROB & 3.791 & & 9.791 & $* * *$ & 3.121 & $*$ \\
\hline M3B & 2.324 & & 6.563 & $* *$ & 1.614 & \\
\hline PM3 & 0.264 & & 13.716 & $* * *$ & 5.671 & $* *$ \\
\hline $\mathrm{BB}$ & 4.323 & $*$ & 19.570 & $* * *$ & 2.100 & \\
\hline $\mathrm{IOB}$ & 0.760 & & 19.359 & $* * *$ & 2.463 & \\
\hline CTG & 2.715 & & 11.326 & $* * *$ & 2.260 & \\
\hline PPD & 0.785 & & 9.436 & $* * *$ & 0.624 & \\
\hline ROL & 1.177 & & 21.392 & $* * *$ & 8.671 & $* * *$ \\
\hline \multicolumn{7}{|c|}{ Mandibular } \\
\hline IM3I & 0.047 & & 11.660 & $* * *$ & 4.847 & $*$ \\
\hline MAL & 0.196 & & 19.658 & $* * *$ & 3.635 & $*$ \\
\hline $\mathrm{MH}$ & 0.909 & & 14.219 & $* * *$ & 1.332 & \\
\hline ML & 9.869 & & 16.184 & $* * *$ & 1.450 & \\
\hline APL & 8.972 & & 0.553 & & 1.062 & \\
\hline
\end{tabular}

than that of the other two groups. Despite the significant differences in the average measurements of most characters in shrews from the three populations, overlaps in the distribution of measurements of characters existed among these three insular populations.

\section{Multivariate analysis}

The canonical discriminant analysis (CDA) was run to examine the degree of separation of the three insular groups, using the following eight characters: BB, CTG, VL, ML, IM3I, M2B, APL and HF. These characters were selected to minimize the value of Wilk's lambda (Kitchener, Schmitt \& Maharadatunkamsi, 1994). The CDA of the three insular groups revealed significant intergroup variation expressed by the two canonical axes, CAN1 and CAN2 (Table 4, Fig. 4). CAN 1 explained $73.9 \%$ of the variance and CAN2, 26.1\%. All individuals were correctly classified to their appropriate island grouping. The plot of CAN1 and CAN2 indicated that the three insular groups were approximately equidistant in discriminate function space. The Taiwan group could be separated from both the Green Island and the Orchid Island groups on CAN1; while the Orchid Island and the Green Island groups could be separated on CAN2.

Values of standardized canonical coefficients given in Table 4 indicated that the greatest proportion of variance on the first axis was expressed by differences in BB (1.421), CTG (-1.237) and IM3I (0.607). ML $(-0.740)$, M2B $(-0.636)$, and CTG $(0.606)$ made the greatest contributions to the variance along the second axis. These results revealed that the Taiwan population has smaller BB/CTG ratio in skull shape than the other two insular populations (Fig. 5), i.e. the shrews of the Taiwan population have narrower brain cases. Student's $t$-test was used to examine the $\mathrm{BB} / \mathrm{CTG}$ ratio of the three insular populations, and the result revealed a significant difference $(P<0.001)$ between Taiwan and the two offshore islands.

The specimens collected in 1933 by Kano from Orchid Island and stored at the National Science Museum, Tokyo (NSMT) were also examined: NSMT 4391, NSMT 4392 and NSMT 4395. NSMT 4391 and 4392 were only skin specimens, and their original labels do not have any measurements. The cranial measurements of NSMT 4395 are smaller than the cranial measurements of most of our specimens, although they are all within the range of our measurements. In addition, NSMT 4395 also has a larger $\mathrm{BB} / \mathrm{CTG}$ ratio (1.105). Therefore, the BB/CTG ratio seems to be an appropriate character to distinguish specimens from Taiwan and Orchid Island.

Results of the CDA indicate that $C$. tadae has three insular forms. Therefore, we propose that they should be described as three insular subspecies: $C$. $t$. tadae (Orchid Island form), C. t. kurodai (Taiwan form), and a new subspecies, C. t. lutaoensis, on Green Island.

\section{SYSTEMATICS}

\section{Crocidura tadae lutaoensis, subsp. nov.}

\section{Holotype}

Adult male, skin and skull, NTU-FS4201, from Green Island, Taitong Hsien, Taiwan; obtained 23 February 1998 by Fang Yin-Ping.

\section{Paratypes}

Seven females and seven males, skins and skulls, NTU-FS4005, NTU-FS4202 to NTU-FS4214; obtained February 1998 by Fang Yin-Ping.

\section{Description}

Size small; dorsal hairs dark reddish brown to dark brown; ventral pelage grey to dark greyish brown; a 
Table 3. Measurements (mm) for external, cranial, mandibular characters of Crocidura tadae from Taiwan, Green Island and Orchid Island (sample size in parenthesis, see Materials and methods for definition of character codes). Values are means, standard deviation (SD) and range (in parenthesis). a-c, Pairwise comparison tested with $t$-test; a, Taiwan $v s$ Green Island; $\mathrm{b}$, Green Island $v s$ Orchid Island; c, Orchid Island $v s$ Taiwan. Significance levels: $*, 0.05>P>0.01 ; * *, 0.01>P>0.001$; $* * *, P<0.001$

\begin{tabular}{|c|c|c|c|c|c|c|c|c|c|}
\hline \multirow[b]{2}{*}{ Character } & \multicolumn{3}{|c|}{ Taiwan $(n=72)$} & \multicolumn{3}{|c|}{ Green Island $(n=15)$} & \multicolumn{3}{|c|}{ Orchid Island $(n=19)$} \\
\hline & Mean & SD & $\mathrm{a}$ & Mean & SD & $\mathrm{b}$ & Mean & SD & $\mathrm{c}$ \\
\hline \multicolumn{10}{|l|}{ External } \\
\hline $\begin{array}{l}\text { HV } \\
(\min -\max )\end{array}$ & $\begin{array}{l}62.442 \\
(55.4-73.3)\end{array}$ & 3.210 & & $\begin{array}{l}63.300 \\
(56.7-69.3)\end{array}$ & 3.753 & $*$ & $\begin{array}{l}59.947 \\
(53.3-64.7)\end{array}$ & 3.204 & $* *$ \\
\hline TV & $\begin{array}{l}48.693 \\
(41.2-58.5)\end{array}$ & 3.774 & $*$ & $\begin{array}{l}51.420 \\
(46.2-57.0)\end{array}$ & 3.297 & $* * *$ & $\begin{array}{l}45.737 \\
(34.2-50.9)\end{array}$ & 3.545 & $* *$ \\
\hline $\mathrm{TH}$ & $\begin{array}{l}0.781 \\
(0.62-0.96)\end{array}$ & 0.067 & & $\begin{array}{l}0.813 \\
(0.74-0.89)\end{array}$ & 0.034 & $* *$ & $\begin{array}{l}0.764 \\
(0.62-0.86)\end{array}$ & 0.059 & \\
\hline $\mathrm{HF}$ & $\begin{array}{l}11.889 \\
(10.5-12.9)\end{array}$ & 0.493 & $*$ & $\begin{array}{l}12.200 \\
(11.5-12.7)\end{array}$ & 0.409 & $* * *$ & $\begin{array}{l}11.442 \\
(10.8-12.0)\end{array}$ & 0.310 & $* * *$ \\
\hline EL & $\begin{array}{l}8.239 \\
(7.0-9.8)\end{array}$ & 0.479 & $*$ & $\begin{array}{l}8.573 \\
(7.9-9.1)\end{array}$ & 0.371 & $* * *$ & $\begin{array}{l}8.016 \\
(7.1-8.6)\end{array}$ & 0.432 & \\
\hline VL & $\begin{array}{l}19.939 \\
(11.9-30.4)\end{array}$ & 3.320 & & $\begin{array}{l}19.753 \\
(16.5-26.2)\end{array}$ & 2.341 & $* * *$ & $\begin{array}{l}14.321 \\
(11.0-18.8)\end{array}$ & 2.065 & $* * *$ \\
\hline VT & $\begin{array}{l}0.409 \\
(0.28-0.54)\end{array}$ & 0.055 & & $\begin{array}{l}0.385 \\
(0.31-0.46)\end{array}$ & 0.396 & $* * *$ & $\begin{array}{l}0.313 \\
(0.25-0.38)\end{array}$ & 0.033 & $* * *$ \\
\hline \multicolumn{10}{|l|}{ Cranial } \\
\hline GLS & $\begin{array}{l}17.689 \\
(16.73-18.61)\end{array}$ & 0.419 & $* *$ & $\begin{array}{l}18.153 \\
(17.45-18.79)\end{array}$ & 0.447 & $* * *$ & $\begin{array}{l}17.245 \\
(16.14-17.91)\end{array}$ & 0.394 & $* * *$ \\
\hline $\mathrm{CL}$ & $\begin{array}{l}16.936 \\
(15.95-17.81)\end{array}$ & 0.422 & $* *$ & $\begin{array}{l}17.435 \\
(16.58-18.11)\end{array}$ & 0.487 & $* * *$ & $\begin{array}{l}16.522 \\
(15.31-17.12)\end{array}$ & 0.412 & $* * *$ \\
\hline PPL & $\begin{array}{l}7.961 \\
(7.34-8.44)\end{array}$ & 0.216 & & $\begin{array}{l}8.086 \\
(7.55-8.63)\end{array}$ & 0.288 & $* * *$ & $\begin{array}{l}7.694 \\
(7.21-7.98)\end{array}$ & 0.191 & $* * *$ \\
\hline IM3S & $\begin{array}{l}7.605 \\
(7.15-8.05)\end{array}$ & 0.223 & $* * *$ & $\begin{array}{l}7.886 \\
(7.65-8.17)\end{array}$ & 0.182 & $* * *$ & $\begin{array}{l}7.485 \\
(7.14-7.84)\end{array}$ & 0.174 & $*$ \\
\hline M2B & $\begin{array}{l}5.249 \\
(5.01-5.50)\end{array}$ & 0.108 & $* * *$ & $\begin{array}{l}5.424 \\
(5.19-5.61)\end{array}$ & 0.134 & $* * *$ & $\begin{array}{l}5.172 \\
(4.92-5.38)\end{array}$ & 0.121 & $*$ \\
\hline ROB & $\begin{array}{l}2.170 \\
(2.00-2.34)\end{array}$ & 0.072 & $* *$ & $\begin{array}{l}2.266 \\
(2.12-2.39)\end{array}$ & 0.089 & $* * *$ & $\begin{array}{l}2.160 \\
(2.06-2.27)\end{array}$ & 0.060 & \\
\hline M3B & $\begin{array}{l}2.105 \\
(1.90-2.25)\end{array}$ & 0.077 & $* * *$ & $\begin{array}{l}2.196 \\
(2.03-2.28)\end{array}$ & 0.069 & $* *$ & $\begin{array}{l}2.117 \\
(1.99-2.23)\end{array}$ & 0.071 & \\
\hline PM3 & $\begin{array}{l}4.402 \\
(4.12-4.73)\end{array}$ & 0.142 & $* * *$ & $\begin{array}{l}4.559 \\
(4.35-4.68)\end{array}$ & 0.098 & $* * *$ & $\begin{array}{l}4.301 \\
(4.12-4.46)\end{array}$ & 4.342 & $*$ \\
\hline $\mathrm{BB}$ & $\begin{array}{l}8.048 \\
(7.60-8.51)\end{array}$ & 0.201 & $* * *$ & $\begin{array}{l}8.449 \\
(8.09-8.81)\end{array}$ & 0.209 & $* * *$ & $\begin{array}{l}8.175 \\
(7.75-8.46)\end{array}$ & 0.197 & $*$ \\
\hline IOB & $\begin{array}{l}3.866 \\
(3.57-4.17)\end{array}$ & 0.136 & $* * *$ & $\begin{array}{l}4.111 \\
(3.92-4.42)\end{array}$ & 0.126 & $* * *$ & $\begin{array}{l}3.855 \\
(3.71-4.06)\end{array}$ & 0.093 & \\
\hline CTG & $\begin{array}{l}7.751 \\
(7.23-8.27)\end{array}$ & 0.216 & & $\begin{array}{l}7.749 \\
(7.39-8.20)\end{array}$ & 0.214 & $* *$ & $\begin{array}{l}7.506 \\
(6.97-7.73)\end{array}$ & 0.179 & $* * *$ \\
\hline PPD & $\begin{array}{l}3.271 \\
(3.08-3.52)\end{array}$ & 0.097 & $* * *$ & $\begin{array}{l}3.394 \\
(3.22-3.54)\end{array}$ & 0.104 & $* * *$ & $\begin{array}{l}3.260 \\
(3.15-3.40)\end{array}$ & 0.079 & \\
\hline ROL & $\begin{array}{l}6.664 \\
(6.25-7.11)\end{array}$ & 0.210 & $* * *$ & $\begin{array}{l}6.954 \\
(6.68-7.29)\end{array}$ & 0.203 & $* * *$ & $\begin{array}{l}6.513 \\
(6.31-6.81)\end{array}$ & 0.137 & $* * *$ \\
\hline \multicolumn{10}{|l|}{ Mandibular } \\
\hline IM3I & $\begin{array}{l}5.240 \\
(4.90-5.74)\end{array}$ & 0.183 & $* * *$ & $\begin{array}{l}5.467 \\
(5.30-5.58)\end{array}$ & 0.099 & $* * *$ & $\begin{array}{l}5.189 \\
(4.97-5.46)\end{array}$ & 0.130 & \\
\hline MAL & $\begin{array}{l}7.197 \\
(6.63-7.71)\end{array}$ & 0.225 & $* * *$ & $\begin{array}{l}7.601 \\
(7.22-7.97)\end{array}$ & 0.293 & $* * *$ & $\begin{array}{l}7.098 \\
(6.69-7.39)\end{array}$ & 0.203 & \\
\hline $\mathrm{MH}$ & $\begin{array}{l}4.021 \\
(3.18-4.30)\end{array}$ & 0.158 & $* * *$ & $\begin{array}{l}4.224 \\
(4.03-4.46)\end{array}$ & 0.121 & $* * *$ & $\begin{array}{l}3.948 \\
(3.79-4.14)\end{array}$ & 0.095 & $*$ \\
\hline ML & $\begin{array}{l}5.338 \\
(5.01-5.80)\end{array}$ & 0.167 & $*$ & $\begin{array}{l}5.468 \\
(5.04-5.76)\end{array}$ & 0.208 & $* * *$ & $\begin{array}{l}5.142 \\
(4.87-5.43)\end{array}$ & 0.152 & $* * *$ \\
\hline APL & $\begin{array}{l}2.007 \\
(1.75-2.29)\end{array}$ & 0.106 & & $\begin{array}{l}2.007 \\
(1.76-2.13)\end{array}$ & 0.090 & & $\begin{array}{l}1.977 \\
(1.62-2.14)\end{array}$ & 0.116 & \\
\hline
\end{tabular}


Table 4. Standardized canonical coefficients based on eight morphometric characters (see Materials and methods for definition of character codes) of Crocidura tadae from Taiwan, Green Island and Orchid Island

\begin{tabular}{lrr}
\hline Character & \multicolumn{1}{c}{ CAN1 } & \multicolumn{1}{c}{ CAN2 } \\
\hline Eigen values & 2.884 & 1.005 \\
Proportion & 0.739 & 0.261 \\
Comulative & 0.739 & 1.000 \\
BB & 1.421 & -0.053 \\
CTG & -1.237 & 0.606 \\
VL & -0.574 & -0.288 \\
ML & -0.418 & -0.740 \\
IM3I & 0.607 & -0.055 \\
M2B & -0.140 & -0.636 \\
APL & 0.134 & 0.597 \\
HF & 0.352 & -0.448 \\
\hline
\end{tabular}

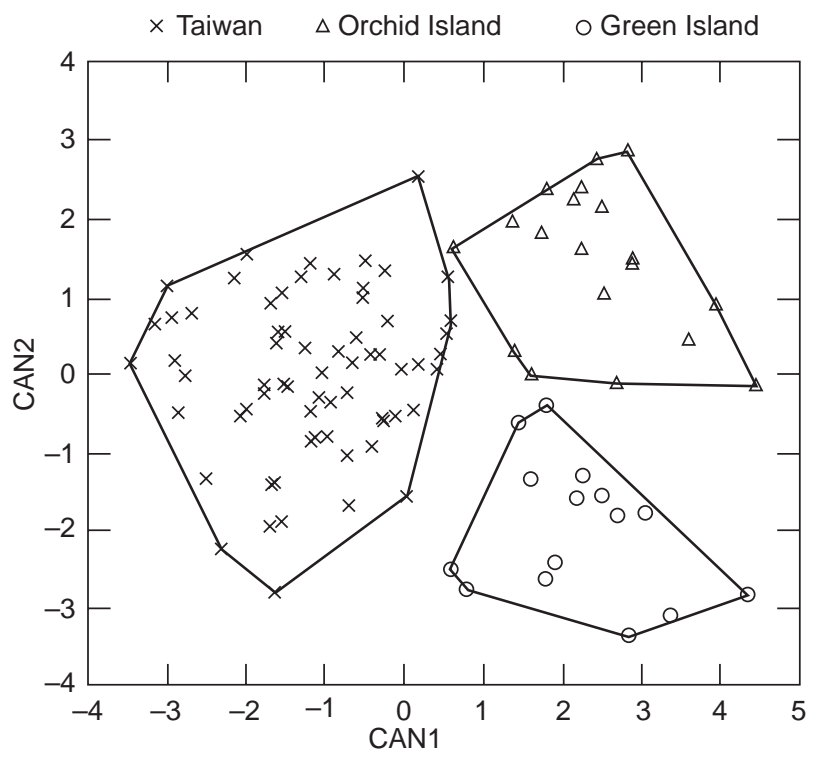

Fig. 4. Scatterplots of discriminant scores on the first (CAN1) and second (CAN2) canonical axes based on eight morphometric characters of Crocidura tadae from Taiwan, Green Island and Orchid Island.

gradual transition in colour along the flanks between dorsum and venter; tail slightly bicoloured, dorsum dark brown, venter lighter dark brown; sparse bristles (3-4 mm long) on the basal one-third to half of the tail; ears large and dark; hind feet large, covered with dark brown hairs dorsally; skull small; condylobasal length long; braincase broad and moderately rounded; rostrum narrow; interorbital region broad; upper tooth row long.

\section{Measurements (mm)}

Measurements of the holotype: head to vent length, 65.8; tail to vent length, 52.6; ear length, 8.4; hind foot length (exclusive of claw), 12.7; distribution of vibrissae

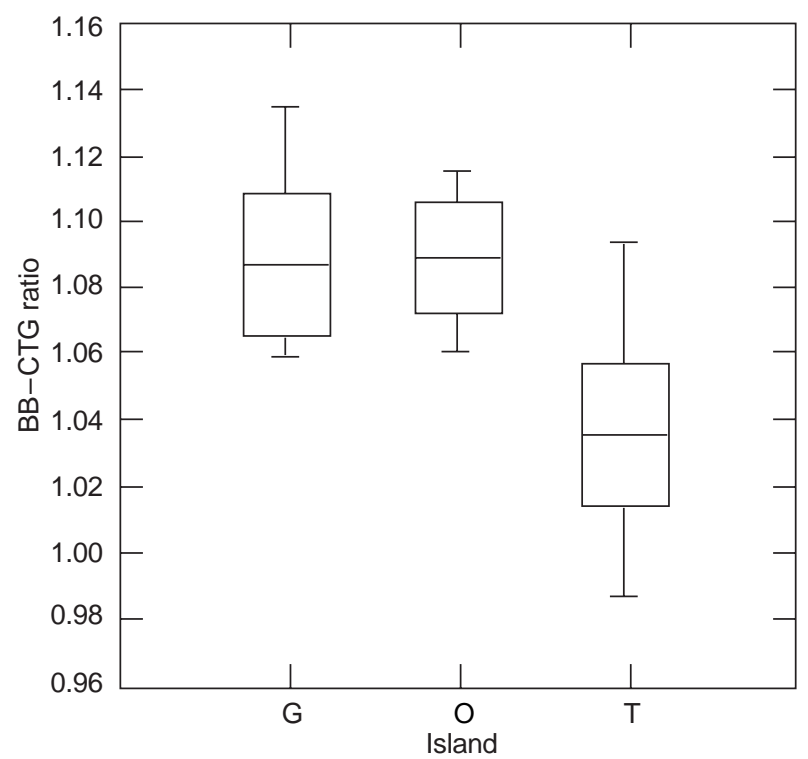

Fig. 5. The ratios of braincase breadth and condyle to glenoid length (BB-CTG ratio) of the three insular populations: G, Green Island; O, Orchid Island; T, Taiwan. Vertical lines, total variation of the samples; rectangles, standard deviation of the mean; horizontal lines, mean.

over the length of the tail, 19.0; weight (g), 6.3; greatest length of skull, 18.51; condylobasal length, 17.64; braincase breath, 8.61; condyle to glenoid length, 7.87; upper tooth row length, 8.09; length of upper molariform teeth, 4.55; lower tooth row length excluding first incisor, 5.58; mandibular length, 7.74. The means and ranges of all the measurements of the paratypes are listed in Table 3.

\section{Comparison}

Crocidura tadae lutaoensis is larger than the other two insular subspecies. There is a general gradation in size from smallest $C$. $t$. tadae through $C$. $t$. kurodai to $C$. $t$. lutaoensis. The dorsal and ventral colour of $C$. $t$. lutaoensis is close to that of $C$. $t$. tadae, but C. t. kurodai shows more variation in colour.

Crocidura t. lutaoensis is slightly bicoloured on the basal part of the tail as in C. t. tadae and C. t. kurodai, however, the dorsal part of the tail in C.t. lutaoensis is darker than that of $C$. $t$. kurodai. The vibrissae of $C$. $t$. lutaoensis as well as $C$. $t$. tadae are usually unicoloured, generally white but with the basal half brown. In C. $t$. kurodai, however, the colour of the vibrissae is variable, i.e. white in some individuals, white distally, or dark brown in others.

The hind feet of Crocidura t. lutaoensis are larger than those of $C$. t. tadae. C. t. lutaoensis also has darker brown hairs covering the dorsum of the hind feet; therefore, their hind feet are relatively darker than those of C. t. tadae and C. t. kurodai. 


\section{Etymology}

Named after Lutao Island.

\section{DISCUSSION}

Chromosomal characterization has been accepted as a useful tool in species identification of Crocidura (Ruedi et al., 1990). Karyotypes of about 50 Crocidura species have been reported (reviewed by Maddalena \& Ruedi, 1994; Ruedi \& Vogel, 1995; Zima, Lukacova \& Macholan, 1998). Substantial karyotypical variation was found among Crocidura species, but little or no variation was found at the intraspecific level. Our results of cytological comparisons revealed that $C$. tadae from three insular populations, i.e. Taiwan, Green Island, and Orchid Island, are similar in conventional and G-banding karyotypes. Comparing all published data of Crocidura karyotypes, only the C. fuliginosa complex (Ruedi et al., 1990) has similar karyotypic characteristics to $C$. tadae, with the same $2 \mathrm{n}$ and FN. However, the autosome of the two groups consisted of unequal pairs of meta- (or submeta-) centric and subtelocentric. This evidence reflected that $C$. tadae has a unique karyotype, which is different from the karyotype of other Crocidura species. Based on chromosomal features, C. kurodai Jameson \& Jones, 1977 is considered as the junior synonym and as a subspecies of $C$. tadae Tokuda \& Kano, 1936.

Although C. tadae shows the same karyotypic characters in three insular populations, they show significant geographic variation in morphological characters. Both univariate and multivariate analyses revealed that the shrews of the Green Island group are larger than those of the Orchid Island group, and shrews of the Taiwan group are intermediate in size but have a larger range in measurements of all the characters. Jameson and Jones (1977) also noted that pelage colour of tadae and kurodai were different. We agreed with their description of C. h. tadae, which has a dark reddish brown dorsum. It is a stable character of the Orchid Island group. However, the dark reddish brown pelage could also be found in shrews of Green Island and Taiwan. Our samples also revealed that great variation existed in each group, and such variation seems to be related to sex, maturity of the animals, and season, because shrews moult in early spring.

Besides the body size and external characters, other differences were also noted among the three groups. For example, the sex ratio of shrews from each insular population was different. The sex ratios on Orchid and Green Island were 1:1. However, at all except one locality (Tong-Pu) of Taiwan, the sex ratio was biased toward males. Cantoni \& Vogel (1989) indicated that the social organization of $C$. russula seemed to be strongly influenced by season. Rychlik (1998), Stockley \& Searle (1998) reviewed the social and mating systems of shrews, and concluded that different sex ratios may be related to different mating strategies. In this study, we collected the shrews mostly in winter, between late autumn and early spring, to minimize differences among populations due to seasonal variation. We found a newborn shrew on Orchid Island in February, and assumed that the shrews of Orchid Island was still breeding. Therefore, we cannot overrule the possibility of seasonal influences on social organization and thus on the sex ratio of these populations.

Our analyses have revealed another special morphological difference. Shrews of the Taiwan population are significantly smaller than those of the Green Island population in most skull measurements, but no significant difference was found in external characters. Contrarily, shrews of the Taiwan population are significantly larger than the Orchid Island population in most external measurements and a few cranial characters, such as greatest length of skull (GLS), postpalatal length (PPL) and condyle to glenoid length (CTG). The trends that different groups may be larger in some measurements but smaller in others have not been reported in other Crocidura groups, such as C. maxi (Kitchener, Hisheh et al., 1994) and C. watasei (Motokawa et al., 1996).

At present, it is difficult to determine the cause of such insular variation. Ecological conditions such as island area, resource competition and limitation, immigrant selection (Heaney, 1978; Kitchener, Schmitt et al., 1994), and historical geographic events (Sara, Valvo \& Zanca, 1990; Motokawa et al., 1996) may play a role. Heaney (1978) concluded that variation in body size of the Asian tricoloured squirrel Callosciurus prevostii is related to island area. Kitchener, Schmitt et al. (1994) reviewed many studies of small mammals and explained variation in body size as related to island area and immigrant selection. In their study, greater distance from the source areas may reduce the effect of immigrant selection and allow the impact of resource limitation on small islands to influence body size negatively. Nevertheless, our data shows an indistinct pattern: Green Island is smaller in land area than Orchid Island (one-third), closer to Taiwan (33 vs $90 \mathrm{~km})$, but has bigger $C$. tadae. Therefore, the ecological condition alone can not explain the variation we observed.

Motokawa et al. (1996) examined the morphometric characters of $C$. watasei from the Ryukyu Archipelago, and suggested that the geographic variation is relevant to historical geographic events. Unfortunately, the geological data regarding to the history of isolation between Taiwan and the two offshore islands are currently inadequate.

Recently, Leuo (1995) and Tsai (1998), respectively, examined some reptiles such as Japarula swinhonis and Gekko hokouensis that have similar distributions to C. tadae. They did not find any significant morphological variation between insular populations. However, they used the Mt-DNA RFLP technique and DNA sequencing to analyse the genetic distance and phylogeny of reptile populations in Taiwan and accessory islands, and found an interesting result. They suggested that the populations of Green Island and Orchid Island 
both originated from south-eastern Taiwan and have a close relationship with each other. One morphological feature of $C$. tadae in our results seems to reveal a similar relationship. The shrews of Green Island and Orchid Island have a more similar skull shape (i.e. larger $\mathrm{BB} / \mathrm{CTG}$ ratio), which is a broader brain case than that of the Taiwan population. Comparisons of the $\mathrm{BB} / \mathrm{CTG}$ ratios of shrews taken from south-eastern Taiwan (Nanjenshan, Locality 7), show that they were significantly different from the shrews of Green Island and Orchid Island. Therefore, morphological features cannot provide further evidence regarding the nature of the origin of the populations on Green Island and Orchid Island.

\section{ACKNOWLEDGMENTS}

We express our sincere thanks to Dr H. Endo (National Science Museum, Tokyo) for allowing YPF to examine the specimens of $C$. tadae, and Dr M. Motokawa for useful discussion of these specimens. Our special thanks to P. D. Jenkins, The Natural History Museum, London; Dr G. Csorba, The Hungarian Natural History Museum; and the anonymous referees for their valuable comments on this manuscript. We thank Drs F. Y. Lin, L. K. Lin, H. Y. Wu, H. T. Yu, and H. W. Yuan for their technical support and advice. In addition, we thank Y. T. Liou, M. H. Wu, S. P. Wu and members of the Wildlife Laboratory, Department of Zoology, National Taiwan University for offering specimens and helping in field collection. This research was partially supported by the Council of Agriculture, R.O.C., grant no. 89 Conservation -07(09).

\section{REFERENCES}

Benn, P. A. \& Perle, M. A. (1986). Chromosome staining and banding techniques. In Human cytogenetics: 57-84. Rooney, D. E. \& Czepulkpwski, B. H. (Eds). Oxford: IRL Press.

Cantoni, D. \& Vogel, P. (1989). Social organization and mating system of free-ranging, greater white-toothed shrews, Crocidura russula. Anim. Behav. 38: 205-214.

Corbet, G. B. \& Hill, J. E. (1992). The mammals of the Indomalayan region: a systematic review. New York: Oxford University Press.

Fang, Y. P., Lee, L. L., Yew, F. H. \& Yu, H. T. (1997). Systematics of white-toothed shrews (Crocidura)(Mammalia: Insectivora: Soricidae) of Taiwan: karyological and morphological studies. J. Zool. (Lond.) 242: 151-166.

Heaney, L. R. (1978). Island area and body size of insular mammals: evidence from the tricoloured squirrel (Callosciurus prevosti) of Southeast Asia. Evolution 32: 29-44.

Hutterer, R. (1993). Order Insectivora. In Mammal species of the world. A taxonomic and geographic reference: 69-130. Wilson, D. E. \& Reeder, D. M. (Eds). Washington, DC: Smithsonian Institution Press.

Jameson, E. W. Jr \& Jones, G. S. (1977). The Soricidae of Taiwan. Proc. Biol. Soc. Wash. 90: 459-482.

Jenkins, P. D. (1976). Variation in Eurasian shrews of the genus Crocidura (Insectivora: Soricidae). Bull. Br. Mus. Nat. Hist. (Zool.). 30(7): 271-309.
Jones, G. S., Lim, B. L. \& Cross, J. H. (1971). A key to the mammals of Taiwan. Chinese J. Microbiol. 4: 267-278.

Kirkland, G. L., Jr \& Sheppard, P. K. (1994). Proposed standard proctocol for sampling small mammal communities. In Advances in the biology of shrews: 277-281. Merritt, J. F., Kirkland, G. L., Jr \& Rose, R. K. (Eds). Carnegie Mus. Nat. Hist. Spec. Publ. No. 18.

Kitchener, D. J., Hisheh, S., Schmitt, L. H. \& Suyanto, A. (1994). Shrews (Soricidae: Crocidura) from the Lesser Sunda Islands, and south-east Maluku, eastern Indonesia. Aust. Mammal. 17: $7-17$.

Kitchener, D. J., Schmitt, L. H. \& Maharadatunkamsi. (1994). Morphological and genetic variation in Suncus murinus (Soricidae: Crocidurinae) from Java, Lesser Sunda islands, Maluku and Sulawesi, Indonesia. Mammalia 58: 433-451.

Kuroda, N. (1952). Mammalogical history of Formosa, with zoogeography and bibliography. Q. J. Taiwan Mus. 5: 262-304.

Lee, M. R. \& Elder, F. F. (1980). Yeast stimulation of bone marrow mitosis for cytogenetic investigation. Cytogenet. Cell Genet. 26: 36-40.

Leuo, K. C. (1995). Phylogeographic relationship of Japalura swinhonis based on analysis of $m t D N A$. Master thesis, National Sun-Yi-Hsien University, Kaohsiung. [In Chinese.]

Maddalena, T. \& Ruedi, M. (1994). Chromosomal evolution in the genus Crocidura (Insectivora: Soricidae). In Advances in the biology of shrews: 335-344. Merritt, J. F., Kirkland, G. L., Jr \& Rose, R. K. (Eds). Carnegie Mus. Nat. Hist. Spec. Publ. No. 18.

Motokawa, M., Hattori, S., Ota, H. \& Hikida, T. (1996). Geographic variation in the watase's shrew Crocidura watasei (Insectivora, Soricidae) from the Ryukyu Archipelago, Japan. Mammalia 60: 243-254.

Rao, K. S. \& Aswathanarayana, N. V. (1978). Karyological analysis of the Horsfield's shrew from peninsular India. J. Hered. 69: 202-204.

Ruedi, M. (1995). Taxonomic revision of shrews of the genus Crocidura from the Sunda Shelf and Sulawesi with description of two new species (Mammalia: Soricidae). Zool. J. Linn. Soc. 115: $211-265$.

Ruedi, M., Maddalena,T., Yong, H. S. \& Vogel, P. (1990). The Crocidura fuliginosa species complex (Mammalia: Insectivora) in Peninsular Malaysia: biological, karyological and genetical evidence. Biochem. Syst. Ecol. 18: 573-581.

Ruedi, M. \& Vogel, P. (1995). Chromosomal evolution and zoogeographic origin of southeast Asian shrew (genus Crocidura). Experientia 51: 174-178.

Rychlik, L. (1998). Evolution of social systems in shrews. In Evoluton of shrews: 347-406. Wojcik, J. M. \& Wolsan, M. (Eds). Bialowieza: Polish Academy of Sciences.

Sara, M., Valvo, M. L. \& Zanca, L. (1990). Insular variation in central Mediterranean Crocidura Wagler, 1832 (Mammalia, Soricidae). Boll. Zool. 57: 283-293.

Seabright, M. (1972). The use of proteolytic enzymes for the mapping of structural rearrangements in the chromosome of man. Chromosoma 36: 204-210.

SPSS (1997). SYSTAT 7.0 programs. Chicago: SPSS Inc.

Stockley, P. \& Searle, J. B. (1998). Shrew mating systems. In Evoluton of shrews: 407-424. Wojcik, J. M. \& Wolsan, M. (Eds). Bialowieza: Polish Academy of Sciences.

Tokuda, M. \& Kano, T. (1936). A bat and a new shrew from Koto-sho (Botel-Tobago). Annot. Zool. Japon. 15: 427-432.

Tsai, C. Y. (1998). Population phylogeny of Gekko hokouensis based on mitochondrial $12 S$ rRNA and cytochrome $b$ sequences. Master thesis, National Sun-Yi-Hsien University, Kaohsiung. [In Chinese].

Zima, J., Lukacova, L. \& Macholan, M. (1998). Chromosomal evolution in shrews. In Evoluton of shrews: 175-218. Wojcik, J. M. \& Wolsan, M. (Eds). Bialowieza: Polish Academy of Sciences. 